\title{
COVID-19 Q\&A: A fish facility down to its core
}

\author{
University College London's Carole Wilson talks to Lab Animal about how she and her fish facility staff have \\ responded to the ongoing coronavirus pandemic.
}

\begin{abstract}
What is your role at your facility, what animals do you care for, and what are they used for?

I am the Technical Head of the fish facility at University College London (UCL) and oversee all operations, which includes the husbandry and welfare of about 70,000 fish, across 6 separate rooms and aquatic systems, varying in size from a 52 multi-racked room holding about 3,000 tanks to individual standalone racks holding approximately 40 tanks; our total capacity is approximately 7,000 tanks.

We primarily hold zebrafish, but also smaller colonies of other species including killifish and cavefish. As a facility, we also provide procedural help, including a genotyping service and a large cryopreservation programme. We provide training including RSB-accredited courses,
\end{abstract} as well as other welfare-based education. We run husbandry and welfare research trials and publish on them. We are currently developing a health monitoring and screening. This is a core facility and this is reflected in the wide variety of research it supports-including developmental biology, angiogenesis, behavioural studies and aging.

\section{How has your facility responded to COVID-19?}

The facility has gone into a lockdown situation, all our research staff have now left-leaving only the core fish facility staff.

Given the current threat involves people potentially going into isolation, we have divided into three discrete teams, which can be subdivided again, if the need arises. We have two teams coming on site every other day, and a third team covering the weekend shifts.

We are currently concentrating on essential husbandry and welfare activities, which is similar to the way in which we provide holiday cover. All our basic husbandry and welfare standard operating procedures are covered: the aquatic systems receive the same maintenance that they always do to function correctly; the fish are fed; the tanks are cleaned; and water quality monitored and adjusted as usual. We are still in contact with all our aquatic equipment suppliers who can provide essential maintenance, in the case of emergency breakdown. We have three separate water

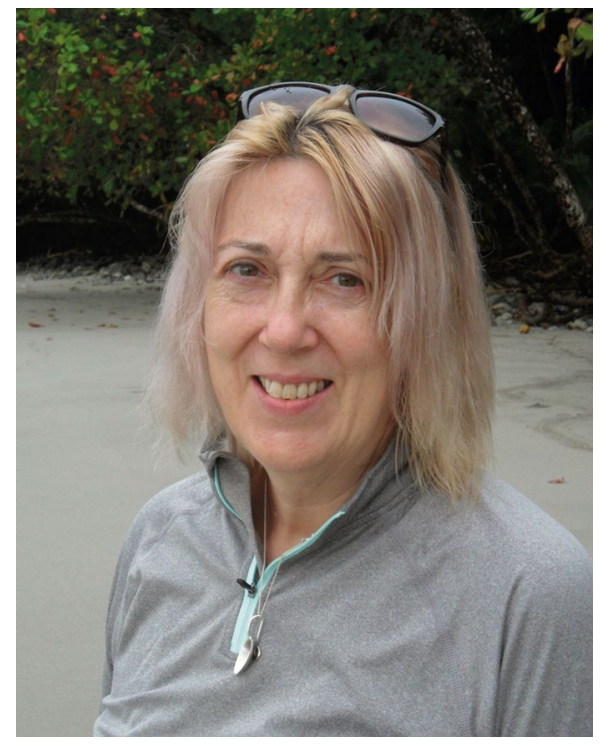

for example, we are no longer teaching and some of our other work will continue offsite (for example, finishing papers and literature searches). All of this can be suspended, depending on people's health and the needs of the essential welfare and husbandry programme.

\section{Did you feel prepared?}

I was asked to prepare an emergency plan for the fish facility about 17 years ago, so at that time I began to consider worse case scenarios-in my mind everything in a fish facility revolves around the provision of excellent quality water; in some emergencies that is a very real problem and one we have faced a couple of times in the past. This is not the problem we are currently facingthis is more about the length of time that the facility is not fully operational, but as we can still receive limited supplies, there is not currently a problem with water or power.

sources and so can share supply between our different systems should the need arise. We also made sure we had about 6 months of supplies before the college closure.

We allowed approximately 10,000 fry to come into the nursery prior to the college closure-this hopefully ensures that researchers have their primary resource when they return to work and gives them good quality stock to work with. We did ask that researchers look at the fish they had and asked that they reduced the numbers of fish they had, either by identifying lines which could go into the cryopreservation programme, older stock that could be replaced by newer fish, and stock that was no longer required for a scientific purpose.

We carry on stock checking and some of us can view our database remotely, so we monitor fish stocks that way as well. This has proved useful to identify strains of fish that could go into the cryopreservation programme, which we hope to reopen in a very modified and smaller form.

On my own project licence I also keep wildtype strains, of which the numbers were reduced as they will not be required for embryo production while researchers are offsite. We also put fry into the nursery to grow up fish for when the facility reopens.

We have suspended some of our non-essential work to concentrate on all husbandry and welfare aspects of the facility;
If that becomes the case then we will have to reduce our stocks further and rely on frozen stocks to replenish strains when we are back up and running.

Additionally, I have always believed in having a very flexible staffing plan. We have a mixture of part- and full-time staff and while everyone has a specialist role, everyone is also trained in the basics-we always have someone of standby to help out. That is really useful in a situation like this.

The biggest challenge was making sure that we had enough staff to cope, and supplies. It felt like a challenge to make sure that people were supplied with key worker letters-that would be the lesson I think should be learnt if there is a next time, but I think this took all the college by surprise.

The other challenge is keeping the team together when people are divided into smaller teams and some are working remotely. We have been holding weekly online meetings where everyone has the opportunity to talk, and I have been following up with individuals about workloads, remote working, and how they are being affected by these extraordinary times.

The lessons I think I have learnt-I am going to encourage people to use the cryopreservation service more, so ensuring that all key lines are preserved if we ever face a situation in the future when we have 
to really reduce down the size of the facility, and that critical work is not lost.

How will things return to normal when the time comes?

I hope that researchers should be able to return to their work as though it was the day before when they left. Obviously, that will depend on exactly how long the facility is shut down for but I anticipate we will be able to provide all of the normal services we usually do from the day the college reopens.

If we start to lose power and/ or water then stocks may have to be reduced further, and it is possible that critical lines will be lost. This may take some time to recover from-it depends how quickly we can, as a community, determine what is critical.

Interviewed by Ellen P. Neff

Published online: 15 April 2020

https://doi.org/10.1038/s41684-020-0533-1

\title{
COVID-19 Q\&A: Collaborating and coordinating keeps a zebrafish facility going
}

\author{
Christine Archer, fish facility coordinator at the University of Colorado Anschutz Medical Campus, walks Lab \\ Animal through her facility's response to the ongoing coronavirus pandemic.
}

What is your role at your facility, what animals do you care for, and what are they used for?

I am the fish facility coordinator at University of Colorado's Anschutz Medical Campus. We have a facility of approximately 25,000 zebrafish. The facility supports diverse biomedical research endeavors in neurobiology, cardiovascular and craniofacial development and more, towards a better understanding of the developmental basis of congenital defects.

How has your facility responded to COVID-19?

Our facility was instructed to shut down on March $17^{\text {th }}$. We had an idea this was coming, so a number of the labs began preparing for contingencies prior to this date. We are running the facility with our two full time animal care professionals, and for the most part the day-to-day animal care is the same as always-with a few exceptions. Only one of the two care staff come in on a day, to reduce risk to everyone. We've reduced to a maintenance feeding, where adults are fed once a day and juvies and subadults get fed twice a day. We've moved to a model where we simply spot change tanks as needed daily, instead of our regular 8-week full change schedule.

We did not have to reduce numbers, though a few labs did reduce some extra broodstock animals, to help lessen the daily check and feeding burden. A number of labs did have to stop everything with regards to embryological work they were in the

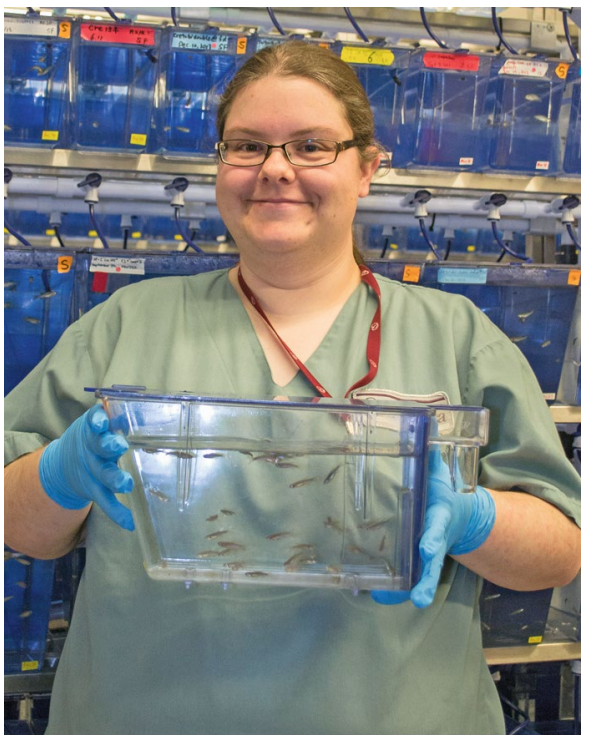

midst of, but the main housing facility was unaffected.

Every day during the shutdown, fish care folks do a morning check in via text and an afternoon debrief via email of the day's happenings and things to consider for the coming days/weeks.

While most fish users were perfectly fine with a 2-week stoppage of all animal work and zebrafish breeding, as the closure extensions have happened we've begun discussing how we should prioritize essential animals to be replaced and how we can maximize breeding to keep the fish stocks in good shape. Likely this will involve cooperation between the fish care team, who have sole access to the facility at this time, and the fish users, who are currently not permitted in the facility. We really want to limit exposure risk to the fish care team, so strict scheduling will need to occur.

\section{Did you feel prepared?}

For the most part, yes. I am actually very new to this position, having only started at the end of 2019. I feel as though I am still getting the hang of everything in this new environment with new colleagues and different ways of working with zebrafish than I am accustomed to. However, I've been working in lab animal care for more than 10 years, with most of that being zebrafish work. I'm also a bit obsessed with being very prepared for all kinds of contingencies, and I've previously written about emergency preparedness for a number of less common pet species.

The fish care team began discussing and preparing for the shutdown more than a week before it was announced. We wrote up our contingency plans as a group, collaboratively with the fish users, and got them approved by the animal care department, ensuring we had a chain of command and care prepared in the event that one or both of the animal care staff were to get sick. We worked extra hours in preparation for a potential shutdown, to make sure everything was stocked, systems were serviced, and as many tanks were changed as possible. I also wrote up very detailed pictorials that would illustrate to just about anyone how to 